\title{
Usabilidade em Aplicativos Móveis Educacionais: Um Conjunto de Heurísticas para Avaliação
}

\author{
Deborah D'Carlo ${ }^{1}$, Glívia Angélica Rodrigues Barbosa ${ }^{1}$, Erica Rodrigues Oliveira ${ }^{2}$ \\ ${ }^{1}$ Centro Federal de Educação Tecnológica de Minas Gerais (CEFET-MG), \\ ${ }^{2}$ Universidade Federal de Minas Gerais (UFMG) \\ deborahdcarlo@gmail.com, gliviabarbosa@decom.cefetmg.br, \\ erica@dcc.ufmg.br
}

\begin{abstract}
This paper aims to present a set of specific heuristics for usability in educational mobile applications. Through assessments with experts and users, it was possible to demonstrate the applicability and relevance of these heuristics for assessing the usability of the educational domain applications.
\end{abstract}

Resumo. Esse trabalho tem como objetivo apresentar um conjunto de heuristicas especificas para usabilidade de aplicativos móveis educacionais. Por meio de avaliações com especialistas e usuários, foi possível evidenciar a aplicabilidade e relevância dessas heurísticas para apreciação da usabilidade de aplicativos do domínio educacional.

\section{Introdução}

A adoção de aplicativos (apps) móveis no contexto educacional vem crescendo e abre espaço para o mobile learning (m-learning), que envolve o uso do dispositivo móvel, sozinho ou em combinação com outras tecnologias de informação e comunicação (TICs) para promover o aprendizado. Diante dessa crescente utilização e dos diferentes perfis de usuários, é relevante garantir que esses aplicativos contemplem requisitos de usabilidade, uma vez que eles possuem objetivos de aprendizado [Kraut, 2013][Knoll, 2014].

Uma forma de caracterizar a usabilidade dos sistemas é a apreciação do modelo de interface e interação desses sistemas, que pode ser conduzida através da aplicação de métodos de avaliação. Dentre os métodos mais difundidos destaca-se a Avaliação Heurística $(\mathrm{AH})$, um método empírico que julga a interface conforme um conjunto de heurísticas (e.g., Consistência e padronização e Design estético e minimalista) que buscam potencializar a usabilidade da interface e da interação [Nielsen, 1994].

Ao apresentar a AH e as 10 heurísticas de usabilidade, Nielsen (1994) alertou para o fato de que sistemas de domínios diferentes demandam por adaptações nas heurísticas de avaliação. No contexto de aplicativos móveis, esse argumento foi reforçado por Bertini et al. (2009) e Nokelainen (2006), que demonstraram em seus estudos problemas de usabilidade que as 10 heurísticas de Nielsen (1994) não permitem caracterizar.

Com o intuito de contornar essas lacunas, Feijó et al. (2013); Neto (2013) e Knoll (2014) propuseram adaptações nas heurísticas de usabilidade para o contexto de aplicações móveis. Contudo, essa ainda é uma questão que apresenta desafios. Isso porque, embora a plataforma destes dispositivos seja a mesma, os aplicativos se diferem, por exemplo, em seus domínios (e.g. aplicativos educacionais, sociais e bancários). Conforme já evidenciado por Knoll (2014), as diferentes categorias de aplicativos podem demandar 
V Congresso Brasileiro de Informática na Educação (CBIE 2016)

Anais do XXVII Simpósio Brasileiro de Informática na Educação (SBIE 2016)

heurísticas de usabilidade específicas para seu domínio, como por exemplo, os aplicativos do domínio educacional [Silveira e Carneiro, 2012].

Motivados por esse cenário, o objetivo deste trabalho é apresentar um conjunto de heurísticas específicas para avaliar e caracterizar a usabilidade de aplicativos móveis educacionais. Para isso identificou-se as heurísticas de usabilidade, existentes na literatura, propostas para aplicativos móveis em geral, e apreciou-se o grau de cobertura das mesmas em relação à usabilidade de aplicativos educacionais. Para cobrir as lacunas observadas na análise anterior, o conjunto de heurísticas específicas para aplicativos móveis educacionais foi proposto e avaliado quanto a sua relevância e aplicabilidade.

Em termos de contribuições práticas, as heurísticas propostas podem ser utilizadas para avaliar aplicativos educacionais existentes e/ou novos, focando na potencialização da usabilidade dos mesmos, além de guiar o projeto de novos aplicativos educacionais. Já em termos científicos, essa pesquisa contribui para avanços na área em relação a um novo conjunto de heurísticas que poderá ser explorado por outros pesquisadores com intuito de validá-lo e/ou delimitar suas vantagens e desvantagens, tanto no projeto quanto para a avaliação de interface de aplicativos móveis educacionais.

\section{Avaliação de Usabilidade em Sistemas Educacionais}

No âmbito das pesquisas voltadas para aplicativos móveis em geral, é possível citar trabalhos como os realizados por Feijó et al. (2013) e Neto (2013) que apresentaram diretrizes de usabilidade para projetar e avaliar esses aplicativos. Os dois conjuntos de diretrizes foram avaliados e, em ambos os trabalhos, argumentou-se que, apesar de contemplarem importantes requisitos de usabilidade para sistemas mobile em geral, as diretrizes apresentam limitações para lidar com as particularidades de cada tipo (i.e., domínio) de aplicativo, e demandam por adaptações para que possam ser utilizadas para avaliar aplicativos de domínios diferentes (e.g., sociais e bancários).

O trabalho conduzido por Knoll (2014) consolidou e apresentou heurísticas de usabilidade para tablets, que foram adaptadas de outras iniciativas presentes na literatura (e.g., Nielsen (1994), Google, Apple e Microsoft). As heurísticas foram avaliadas quanto a sua cobertura para caracterizar a usabilidade de aplicativos móveis e os resultados indicaram que, embora elas sejam mais adequadas, quando comparadas às heurísticas de Nielsen (1994), as heurísticas propostas não foram capazes de cobrir todas as particularidades de usabilidade das diferentes categorias desses aplicativos.

No âmbito de trabalhos direcionados especificamente para aplicativos móveis educacionais não foram encontradas iniciativas similares. Porém, é possível identificar trabalhos como Nokelainen (2006), Oliveira et al. (2009), Silveira e Carneiro (2012) e Kraut (2013), que apresentam e discutem critérios e/ou requisitos de usabilidade para construir e avaliar sistemas educacionais, de modo que esses sistemas de fato apoiem seus usuários no processo de aprendizado. Tais critérios e/ou requisitos caracterizam importantes aspectos de usabilidade que devem ser contemplados nesse tipo de sistema e, por isso, podem ser usados como insumo para criar heurísticas de usabilidade voltadas para aplicativos móveis educacionais [Silveira e Carneiro, 2012] [Feijó et al. 2013].

Este trabalho se difere dos demais, por apresentar um conjunto de heurísticas específico para avaliar e caracterizar a usabilidade de aplicativos móveis educacionais, 
que visa cobrir as lacunas que as heurísticas existentes na literatura não conseguem avaliar em relação à usabilidade desse domínio de aplicação. Além disso, o conjunto de heurísticas proposto poderá ser utilizado tanto para aplicativos educacionais já desenvolvidos, quanto para aplicativos em fase de desenvolvimento (i.e., projeto).

\section{Metodologia}

O presente trabalho foi conduzido em três etapas. Inicialmente foi realizado um levantamento bibliográfico para identificar a existência de heurísticas de usabilidade propostas especificamente para aplicativos móveis. A partir desse levantamento, foi feita uma apreciação crítica da aderência das heurísticas existentes para o domínio de aplicativos móveis educacionais.

A partir dos resultados da apreciação crítica e dos critérios de usabilidade para sistemas educacionais propostos por Nokelainen (2006) e Silveira e Carneiro (2012), foi proposto um conjunto de heurísticas específicas para apreciar a usabilidade de aplicativos móveis educacionais. As etapas de análise da aderência das heurísticas existentes na literatura e a proposição das heurísticas específicas foram realizadas com o auxílio de um especialista em educação (i.e., um pedagogo que atua há mais de sete anos na área e que utiliza tecnologias móveis em suas práticas pedagógicas).

Por fim, a última etapa consistiu em avaliar o conjunto de heurísticas proposto sob duas perspectivas. A primeira buscou verificar, por meio da aplicação de um questionário online, a relevância das heurísticas para avaliar a usabilidade de aplicativos móveis educacionais na perspectiva de especialistas em Interação Humano-Computador (IHC) e Educação. Em seguida, as heurísticas foram avaliadas quanto a sua aplicabilidade para caracterizar a usabilidade dos aplicativos educacionais. Para isso foi conduzido um estudo de caso comparativo em dois aplicativos móveis educacionais, o Nota10 e Passei!ENEM. Esses aplicativos estão entre os 5 primeiros no ranking de downloads e são considerados úteis no contexto educacional brasileiro, por auxiliarem os usuários na preparação do Exame Nacional do Ensino Médio (Enem) [Google Play, 2015]. A seguir, cada etapa da metodologia será detalhada e seus principais resultados serão apresentados e discutidos.

\section{Aderência das Heurísticas da Literatura ao Contexto Educacional}

A primeira etapa deste trabalho consistiu em uma revisão bibliográfica para levantar heurísticas propostas especificamente para caracterizar a usabilidade em aplicativos móveis em geral, e verificar a aderência dessas heurísticas para o domínio de aplicativos móveis educacionais. As buscas foram realizadas no Google Scholar ${ }^{1}$ e nos principais repositórios de pesquisas relacionados à área de Computação, IEEE Xplore ${ }^{2}$, ACM Digital Library ${ }^{3}$, Springer ${ }^{4}$ e HCI Bib ${ }^{5}$. A string de pesquisa contemplava os seguintes termos (em português e, respectiva, tradução em inglês): usabilidade, aplicativos móveis, heurísticas, diretrizes e requisitos.

\footnotetext{
${ }^{1}$ Google Scholar - https://scholar.google.com.br/

${ }^{2}$ IEEE Xplore - http://ieeexplore.ieee.org/Xplore/home.jsp

${ }^{3}$ ACM Digital Library - http://dl.acm.org/

${ }^{4}$ Springer - http://www.springer.com/br/

${ }^{5} \mathrm{HCI}$ Bib - http://hcibib.org/
} 
V Congresso Brasileiro de Informática na Educação (CBIE 2016)

Anais do XXVII Simpósio Brasileiro de Informática na Educação (SBIE 2016)

A partir dessa revisão foram identificados três conjuntos de heurísticas de usabilidade para aplicativos móveis e, uma vez que uma mesma heurística poderia ser proposta por diferentes autores, foi necessário agrupar as heurísticas encontradas. Esse agrupamento foi realizado por um especialista em IHC, profissional da área com mais de cinco anos de experiência, de modo que um conjunto unificado de heurísticas fosse gerado. Esse conjunto é apresentado na Tabela 1. Neste trabalho essas heurísticas, identificadas a partir da literatura, foram denominadas "heurísticas genéricas para aplicativos móveis" (HGs), uma vez que elas não foram propostas para um domínio específico desses aplicativos.

Tabela 1: Heurísticas Genéricas (HGs) para Usabilidade de Aplicativos Móveis

\begin{tabular}{|l|l|}
\hline \multicolumn{1}{|c|}{ Cod. Nome } & \multicolumn{1}{c|}{ Autores } \\
\hline HG1. Feedback imediato e fácil de ser notado & Feijó et. al. (2013), Neto (2013) e Knoll (2014) \\
\hline HG2. Adequação de mensagem à funcionalidade e ao usuário & Feijó et. al. (2013) e Knoll (2014) \\
\hline HG3. Visibilidade e acesso fácil às informações existentes & Feijó et. al. (2013) e Knoll (2014) \\
\hline HG4. Consistência e padrões & Feijó et. al. (2013), Neto (2013) e Knoll (2014) \\
\hline HG5. Prevenção de erros e retomada ao último estado estável & Feijó et. al. (2013) e Knoll (2014) \\
\hline HG6. Minimização da carga de memória do usuário & Feijó et. al. (2013), Neto (2013) e Knoll (2014) \\
\hline HG7. Facilidade de entrada de dados & Feijó et. al. (2013), Neto (2013) e Knoll (2014) \\
\hline HG8. Facilidade de acesso às funcionalidades & Feijó et. al. (2013), Neto (2013) e Knoll (2014) \\
\hline HG9. Ajuda e documentação & Feijó et. al. (2013) e Knoll (2014) \\
\hline HG10. Bom aproveitamento do espaço da tela & Knoll (2014) \\
\hline HG11. Regue incentivo & Feijó et. al. (2013) \\
\hline HG12. Conheça-me & Feijó et. al. (2013) e Knoll (2014) \\
\hline
\end{tabular}

Não foram encontradas heurísticas que abordassem aspectos específicos de usabilidade para os diferentes domínios de aplicativos, sobretudo para os aplicativos educacionais. Sendo assim, foi verificada a aderência das heurísticas genéricas (HGs) para caracterizar a usabilidade dos aplicativos desse domínio.

\subsection{Aderência das Heurísticas Genéricas para o Domínio Educacional}

A análise de aderência das HGs, agrupadas na Tabela 1, para avaliar a usabilidade de aplicativos educacionais foi conduzida por 2 especialistas em IHC, com o apoio de um especialista em Educação. Nesta fase, avaliou-se o aplicativo Nota10, seguindo os passos da Avaliação Heurística, guiada pelas HGs de usabilidade para aplicativos móveis.

Para apreciar o grau de cobertura dessas heurísticas, buscou-se verificar: (1) o total de problemas reportados; (2) o volume de heurísticas violadas e (3) a adequação das heurísticas para avaliar a usabilidade de aplicativos móveis educacionais. Tanto na fase de inspeção quanto na consolidação, o especialista em educação apoiou os avaliadores na identificação de problemas e na justificativa. A avaliação foi realizada no mês de agosto de 2015. Foram considerados os seguintes cenários: (1) fazer questões aleatórias, (2) realizar prova completa, (3) criar simulado e (4) avaliar desempenho. Esses cenários foram escolhidos por representarem as principais funcionalidades do aplicativo Nota 10.

Concluída a avaliação, foram encontrados 11 problemas de usabilidade e $67 \%$ das doze heurísticas foram violadas pelo menos uma vez pelo aplicativo. A avaliação também indicou que 33\% das heurísticas não foram violadas. Isso pode caracterizar que elas estão presentes na interface, como é o caso das heurísticas HG5, HG9 e HG11, ou 
V Congresso Brasileiro de Informática na Educação (CBIE 2016)

Anais do XXVII Simpósio Brasileiro de Informática na Educação (SBIE 2016)

que elas não são indicadas (i.e., necessárias) para avaliar esse domínio de aplicativo, como é o caso da heurística HG12.

Com essa avaliação foi possível reforçar a hipótese de que, apesar das HGs contemplarem importantes critérios de usabilidade de aplicativos móveis em geral, elas não endereçam problemas específicos que podem ocorrer durante a interação com aplicativos educacionais. Isso porque, baseado nessa avaliação e na opinião do especialista em educação, durante a interação com o aplicativo, foram identificados potenciais problemas de usabilidade que poderiam impactar no processo de aprendizado utilizando o aplicativo. Porém esses problemas não puderam ser mapeados (i.e., não havia uma equivalência) para nenhuma $\mathrm{HG}$ existente.

Um desses problemas corresponde ao fato de que, embora o aplicativo ofereça a opção de realizar provas completas ou aleatórias, as respostas fornecidas no modo aleatório não são contabilizadas na análise do desempenho do usuário. Tal problema compromete a consolidação do aprendizado. Embora o problema exista, não seria possível associá-lo a uma HG existente. Nesse caso, seria necessário criar uma heurística específica para o domínio educacional, como por exemplo, "Oferecer diferentes experimentações de aprendizado, incluindo os erros", e indicar que o referido problema estava violando a heurística proposta. Constata-se, então, que a utilização das HGs para avaliar aplicativos educacionais poderia resultar em uma análise superficial, focada em aspectos de interface, que deixaria de considerar pontos importantes durante o processo de interação com esse tipo de aplicativo.

\section{Heurísticas de Usabilidade Específicas para Apps Móveis Educacionais}

Para a criação das heurísticas específicas, foram consideradas três perspectivas: (1) as heurísticas genéricas para aplicativos móveis (HG), por descreverem requisitos básicos de usabilidade para a interface de qualquer tipo de aplicativo; (2) os requisitos de usabilidade de sistemas educacionais listados por Nokelainen (2006) e Silveira e Carneiro (2012); e (3) o conhecimento do especialista em educação. Essas perspectivas atuaram como insumo para adaptar e criar as heurísticas específicas para aplicativos móveis educacionais.

A Tabela 2 mostra as heurísticas de usabilidade específicas (HE) para aplicativos móveis educacionais. Nesta tabela é apresentado o código e o nome de cada heurística e sua origem. A origem foi separada entre três grupos: (1) Heurística Nova - que representa heurísticas integralmente criadas para o contexto de aplicativo educacional; (2) Heurística Adaptada - representa heurísticas genéricas adaptadas pelos autores para o contexto educacional; e (3) Heurística Reutilizada - indica que a heurística não sofreu alteração, e foi retirada da Tabela 1 das HGs.

Tabela 2: Heurísticas Específicas para Usabilidade de Aplicativos Móveis Educacionais

\begin{tabular}{|l|l|}
\hline \multicolumn{1}{|c|}{ Cód. Nome } & \multicolumn{1}{c|}{ Origem } \\
\hline HE1. Consistência e bom aproveitamento do espaço da tela & Heurística Adaptada \\
\hline HE2. Disponibilidade (Acesso de qualquer lugar) e Portabilidade & Heurística Nova \\
\hline HE3. Facilidade de acesso às funcionalidades & Heurística Adaptada \\
\hline HE4. Resposta Imediata e Visibilidade & Heurística Adaptada \\
\hline HE5. Adequação de mensagem à funcionalidade e ao usuário & Heurística Adaptada \\
\hline HE6. Prevenção de erros e recuperação rápida ao último estado estável & Heurística Adaptada \\
\hline HE7. Aprendizado orientado a um objetivo & Heurística Nova \\
\hline
\end{tabular}


V Congresso Brasileiro de Informática na Educação (CBIE 2016)

Anais do XXVII Simpósio Brasileiro de Informática na Educação (SBIE 2016)

\begin{tabular}{|l|l|}
\hline HE8. Aprendizagem e Recordação no processo de Interação & Heurística Nova \\
\hline HE9. Controle de aprendizado e Flexibilidade & Heurística Adaptada \\
\hline HE10. Disponibilizar diferentes experimentações de aprendizado, incluindo erros & Heurística Nova \\
\hline HE11. Aprendizado cooperativo/colaborativo & Heurística Nova \\
\hline HE12. Motivação & Heurística Adaptada \\
\hline HE13. Consolidação de novos aprendizados (Aplicabilidade) & Heurística Nova \\
\hline HE14. Ajuda e documentação & Heurística Reutilizada \\
\hline
\end{tabular}

\section{Análise da Relevância e Aplicabilidade das Heurísticas Propostas}

Com o intuito de ressaltar as contribuições das heurísticas propostas neste trabalho para o contexto de usabilidade dos aplicativos móveis educacionais o conjunto, aqui proposto, foi avaliado e analisado quanto a sua relevância e aplicabilidade. Essas avaliações são detalhadas e discutidas a seguir.

\subsection{Relevância das Heurísticas Propostas sob a Perspectiva de Especialistas}

A relevância do conjunto de heurísticas propostas foi avaliada por especialistas em IHC e Educação, através de um questionário online com as quatorze HEs. Nesta avaliação, cada participante deveria indicar o grau de relevância das mesmas de acordo com a seguinte escala: Fortemente Relevante; Relevante; Pouco Relevante e Irrelevante. A aplicação do questionário ocorreu durante o mês de setembro de 2015, durante 2 semanas. $\mathrm{O}$ mesmo foi divulgado por e-mails para os pesquisadores e profissionais das áreas, com pelo menos 05 anos de experiência. Ao todo 27 especialistas participaram.

Os resultados obtidos permitiram constatar que houve uma aceitação de mais de $90 \%$ das heurísticas propostas. Sendo que, desse percentual de aceitação, 58\% das heurísticas foram consideradas como 'Fortemente Relevantes' e 42\% como 'Relevantes' ao contexto. Ao analisar o grau de relevância por heurística, observou-se que as heurísticas integralmente novas possuem, pelo menos, 70\% de aprovação considerando as opções 'Fortemente Relevante' e 'Relevante'. A Figura 1 apresenta esses resultados.

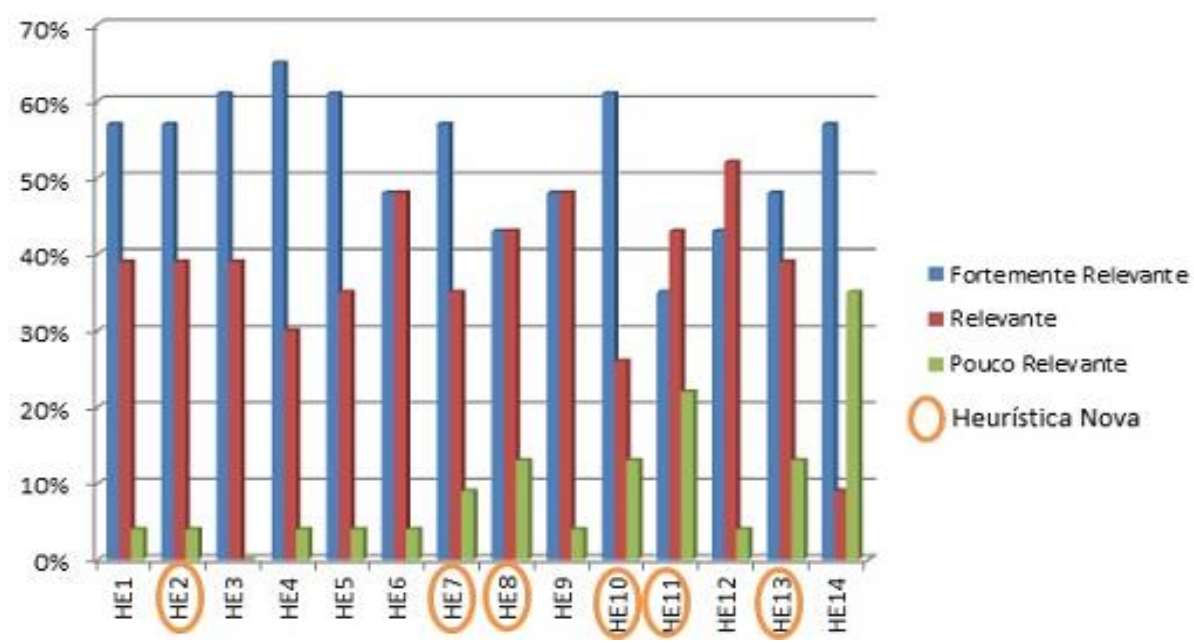

Figura 1: Relevância por Heurística

Algumas considerações foram feitas pelos especialistas em IHC e Educação sobre as heurísticas propostas. Por exemplo, um dos especialistas afirmou que encontrar aplicativos que atendam à heurística "HE2 Disponibilidade - Acesso de qualquer lugar e portabilidade" seria um diferencial de usabilidade desses aplicativos, uma vez que a 
V Congresso Brasileiro de Informática na Educação (CBIE 2016)

Anais do XXVII Simpósio Brasileiro de Informática na Educação (SBIE 2016)

mobilidade do dispositivo favorece o processo de aprendizado em qualquer lugar, sem dependência de conexões com a internet.

Além disso, segundo os especialistas, o índice de aceitação da heurística "H7Aprendizado orientado a um objetivo" foi associado ao argumento apresentado por Kraut (2013). Segundo o autor, uma vez que as metas e objetivos são delineados, eles podem ser sequenciados em hierarquias de aprendizagem que, em geral, representam uma progressão de ensino crescente e facilita no processo de aprendizado.

\subsection{Aplicabilidade das Heurísticas para Avaliar Aplicativos Móveis Educacionais}

Para analisar a aplicabilidade do conjunto de heurísticas específicas para usabilidade de aplicativos educacionais, realizou-se uma análise comparativa. Nessa análise, os aplicativos Nota10 e o Passei!ENEM foram avaliados por dois grupos de especialistas em IHC, seguindo os passos da Avaliação Heurística. Porém, um grupo de especialistas foi guiado pelo conjunto de heurísticas genéricas para aplicativos móveis, que será referenciado como $\mathrm{HG}$, e o outro grupo pelas heurísticas propostas neste trabalho, que será referenciado como HE.

Ao fim das avaliações, os resultados foram contrastados, observando o volume de problemas e se a avaliação guiada pelas HE apontou problemas que só poderiam ser identificados pelas heurísticas propostas neste trabalho. Posteriormente, comparou-se o indicador de usabilidade obtido com a avaliação guiada pelas HEs propostas e o indicador gerado a partir de testes com usuários realizados no Passei!ENEM.

\subsubsection{Cobertura das Heurísticas para Avaliar o Aplicativo Nota 10}

Inicialmente, para comparar a eficiência das heurísticas, foram contrastados os resultados da avaliação do aplicativo Nota10, guiada pelas HGs (seção 4.1.), com os resultados da avaliação guiada pelas HEs, também no app Nota10. A avaliação guiada pelas HEs foi realizada por 4 especialistas, durante o mês de outubro de 2015, considerando os mesmos cenários usados durante a análise do aplicativo com as HGs.

Ao comparar os resultados, foi possível observar que o número de problemas identificados por cada conjunto de heurísticas, HGs e HEs, foi igual, ou seja, 11 problemas cada. Contudo, embora a quantidade de problemas tenha sido a mesma, a análise guiada pelas HEs apresentou um maior volume de heurísticas violadas, quando comparadas às HGs. Isso porque as HEs para aplicativos educacionais foram capazes de apontar 19\% de violações a mais, quando comparadas com as HGs.

Em relação ao grau de cobertura das HEs para apreciar a usabilidade de aplicativos educacionais, todas foram consideradas adequadas para avaliar o aplicativo Nota10. Isso porque $86 \%$ das heurísticas propostas foram violadas na interface, pelo menos uma vez, e as 14\% restantes (e.g., HE11 e HE13), embora não tenham sido violadas, foram apontadas pelos avaliadores como presentes na interface. Portanto, não foram indicadas como fora do contexto da aplicação. Já no caso das HGs, $67 \%$ foram violadas, e das $33 \%$ restantes, a HG12, por exemplo, foi considerada pelos especialistas como não adequada para o contexto da avaliação.

Esses dados reforçam a relevância do conjunto proposto, já indicado como positivo pelos especialistas, uma vez que, conforme demonstrado pelos resultados, avaliando o aplicativo Nota10 pelas HGs não seria possível identificar potenciais violações e estratégias de usabilidade reportados pelas HEs. 
V Congresso Brasileiro de Informática na Educação (CBIE 2016)

Anais do XXVII Simpósio Brasileiro de Informática na Educação (SBIE 2016)

\subsubsection{Cobertura das Heurísticas para Avaliar o Aplicativo Passei! ENEM}

Para melhor apreciar a eficiência das heurísticas propostas, um novo estudo de caso comparativo foi realizado com o aplicativo Passei!ENEM. A avaliação foi conduzida, seguindo os passos da Avaliação Heurística, por quatro grupos, formados por 4 avaliadores cada. Desses grupos, dois ficaram responsáveis pela avaliação guiada pelas HGs, e os outros dois inspecionaram a interface através das HEs.

Finalizadas as avaliações, os resultados foram consolidados e contrastados, observando-se o volume de problemas, bem como o percentual de heurísticas violadas. De forma complementar, conforme sugerido por Knoll (2014), foi gerado um indicador, baseado no percentual de heurísticas violadas, para fornecer uma classificação geral de usabilidade para o aplicativo avaliado. A Tabela 3 apresenta as classificações propostas.

Tabela 3: Classificação de Usabilidade [Knoll, 2014]

\begin{tabular}{|l|l|}
\hline Percentual de Heurísticas Violadas & Usabilidade \\
\hline Maior que $75 \%$ do total & Péssima \\
\hline Maior que $50 \%$ e menor ou igual a $74 \%$ do total & Ruim \\
\hline Maior que $25 \%$ e menor ou igual a $50 \%$ do total & Boa \\
\hline Menor ou igual a $25 \%$ do total & Muito boa \\
\hline
\end{tabular}

A avaliação foi realizada na terceira semana do mês de outubro de 2015. Durante a avaliação foram considerados os seguintes cenários: (1) fazer questões de uma determinada matéria a ser escolhida pelo avaliador; (2) responder uma prova rápida; e (3) consultar ranking. Esses cenários foram escolhidos por representarem as principais funcionalidades do aplicativo. Para melhor comparação, os resultados são apresentados na Tabela 4.

Tabela 4: Resultado da Avaliação do Passei!ENEM

\begin{tabular}{|c|c|c|c|}
\hline & $\begin{array}{c}\mathrm{N}^{\circ} \text { de } \\
\text { problemas }\end{array}$ & $\begin{array}{c}\text { \% de heurísticas } \\
\text { violadas }\end{array}$ & $\begin{array}{c}\text { Classificação da } \\
\text { Usabilidade }\end{array}$ \\
\hline HG & 13 & $71 \%$ & Ruim \\
\hline HE & 10 & $79 \%$ & Péssima \\
\hline
\end{tabular}

Através desses dados, observou-se que, embora os avaliadores guiados pelas HGs tenham identificado um número maior de problemas, o percentual de heurísticas violadas na avaliação realizada com as HEs é superior em relação violações das HGs. Logo, o grau de cobertura das HEs é maior. Isso porque, do ponto de vista da avaliação realizada com as HGs a usabilidade do aplicativo Passei!ENEM foi considerada como 'Ruim'. Já a avaliação guiada pelas HEs classificou a usabilidade do aplicativo como 'Péssima', o que pode indicar que as HEs foram capazes de caracterizar melhor os potenciais problemas de usabilidade do aplicativo.

A próxima fase da avaliação do conjunto de HE proposto consistiu em realizar um teste de usabilidade com usuários e contrastar o resultado obtido com os da Avaliação Heurística. Essa avaliação tinha como objetivo avaliar a usabilidade do aplicativo Passei!ENEM, na opinião dos usuários. Esperava-se verificar se, de fato, os potenciais problemas levantados pelos especialistas, com as HEs, são vivenciados pelos usuários em tempo de interação.

\subsubsection{Avaliação sob a Perspectiva dos Usuários}

O teste de usabilidade foi realizado com cinco usuários, estudantes do ensino médio, com idades entre 15 e 22 anos. Os usuários foram convidados a realizar três tarefas no 
V Congresso Brasileiro de Informática na Educação (CBIE 2016)

Anais do XXVII Simpósio Brasileiro de Informática na Educação (SBIE 2016)

aplicativo: (1) fazer prova com questões aleatórias; (2) fazer prova de matéria específica e (3) reiniciar o aplicativo. Durante os testes, os avaliadores verificaram se as tarefas foram concluídas, com ou sem erros, ou ainda se não foram concluídas.

Durante a avaliação foi possível observar que, apesar de concluírem todas as tarefas, os cinco usuários executaram a tarefa $1 \mathrm{com}$ erros. Apenas um usuário concluiu a tarefa 2 sem erros e dois usuários executaram a tarefa 3 com erros. Constata-se então que a maioria dos usuários encontrou dificuldades para executar as tarefas relacionadas à realização de provas. $\mathrm{O}$ relato dos usuários quanto a essas dificuldades revela que o aplicativo não deixa claro que existam questões aleatórias na opção "Provas Rápidas". Além disso, eles reportaram dificuldades como: (1) passar para a próxima questão; (2) dúvidas quanto aos elementos de interface (e.g., ícones) utilizados na aplicação; e (3) ausência de um feedback mais efetivo.

Finalizada as tarefas, os usuários foram convidados a indicar o nível de satisfação ao interagir com o aplicativo avaliado e, posteriormente, avaliá-lo de acordo com as HEs para caracterizar a usabilidade de aplicativos educacionais. Em relação à satisfação, dentre as opções, $60 \%$ dos usuários indicaram como ruim ou péssima. No que se refere ao julgamento da interface em relação às HEs para aplicativos móveis educacionais, apenas duas heurísticas (HE3 e HE8) foram consideradas presentes no aplicativo por todos os usuários. Isto é, na opinião de pelo menos um dos usuários, $86 \%$ das heurísticas propostas neste trabalho foram violadas pelo aplicativo Passei!ENEM.

\subsection{Discussão dos Resultados}

Para concluir a apreciação da relevância e aplicabilidade das heurísticas propostas, os indicadores de usabilidade gerados a partir da avaliação com especialistas guiada pelas HEs e o teste dos usuários foram contrastados.

Ao mapear o percentual de violação das HEs, na opinião dos usuários, que apontou para $86 \%$ de violações, o aplicativo Passei!ENEM foi classificado como "Péssimo". De forma análoga, a avaliação de usabilidade guiada pelas HEs, realizada por especialistas, também reportou esse resultado, uma vez que indicou $79 \%$ de violações, classificando o aplicativo como "Péssimo".

Conclui-se, então, que a avaliação do Passei!ENEM, guiada pelas HEs, ao contrário das HGs, obteve o mesmo indicador de usabilidade que os usuários indicaram, 'Péssimo'. Isso demonstra que as HEs estão aptas a serem utilizadas como insumo para avaliação de usabilidade dos aplicativos móveis educacionais.

\section{Conclusões e Direções Futuras}

Este trabalho apresentou um novo conjunto de heurísticas para apreciação da usabilidade de aplicativos móveis educacionais, incorporando tanto aspectos gerais de aplicativos móveis quanto requisitos de usabilidade específicos do domínio educacional. A avaliação do conjunto proposto buscou apreciá-lo sob diferentes perspectivas, o que permitiu demonstrar sua relevância diante de especialistas das áreas relacionadas, bem como sua aplicabilidade para caracterizar a usabilidade de aplicativos educacionais.

Esse tipo de pesquisa é relevante porque as heurísticas propostas poderão ser utilizadas para avaliar aplicativos educacionais existentes e/ou novos, focando na potencialização da usabilidade, além de guiar no projeto de novos aplicativos 
V Congresso Brasileiro de Informática na Educação (CBIE 2016)

Anais do XXVII Simpósio Brasileiro de Informática na Educação (SBIE 2016)

educacionais. Em termos científicos, essa pesquisa contribui para avanços na área de IHC, uma vez que um novo conjunto de heurísticas poderá ser explorado por outros pesquisadores com intuito de validá-lo e/ou delimitar suas vantagens e desvantagens, tanto no projeto quanto para a avaliação de interface de aplicativos móveis educacionais.

Como trabalhos futuros, almeja-se validar o conjunto de heurísticas proposto utilizando outros aplicativos educacionais, com diferentes focos de aprendizagem. Além disso, pode-se fazer uso das heurísticas para projetos de interfaces de aplicativos educacionais, explorando o conjunto além do seu uso para avaliação.

\section{Referências}

Bertini, E., Catarci, T.; Dix, A.; Gabrielli, S.; Kimani, S.; Santucci, G. 2009. Appropriating heuristic evaluation for mobile computing. Int. J. Mobile Hum. Comput. Interact. v. 1, n. 1, p. 20-41.

de Oliveira, Erica R.; Gisleide AM Werneck; Raquel O. Prates. 2009. Investigando as Contribuições do Uso de Scaffolds no Domínio Educacional. In Proc of. XX SBIE 2009.

Feijó, V. C.; Gonçalves, B. S.; Gomez, L. S. R. 2013. Heurística para avaliação de usabilidade em interfaces de aplicativos smartphones: Utilidade, produtividade e imersão. Design \& Tecnologia, v. 3, n. 06.

Google Play. 2015. Mobile App Categories. Disponível em: https://goo.gl/AwVIrU. Acesso em Out. 2015.

Knoll, R. C. 2014. Desenvolvimento de heurísticas de usabilidade para tablets. Caderno de Estudos Tecnológicos, v. 2, n. 1, p. 93-109.

Kraut, R. 2013. UNESCO policy guidelines for mobile learning. France: UNESCO.

Neto, O. J. M. 2013. Usabilidade da interface de dispositivos móveis: heurísticas e diretrizes para o design. Tese (Doutorado) - Universidade de São Paulo.

Nielsen, J. 1994 Usability inspection methods. In: ACM. Conference companion on Human factors in computing systems. p. 413-414.

Nokelainen, P. 2006 An empirical assessment of pedagogical usability criteria for digital learning material with elementary school students. Educational Technology \& Society, v. 9, n. 2, p. 178-197.

Silveira, M.S. and Carneiro, M.L.F., 2012, November. Diretrizes para a Avaliação da Usabilidade de Objetos de Aprendizagem. In Proc of. SBIE 2012 (Vol. 23, No. 1). 